\title{
sequence of targeting vector
}

GTGAACCATCACCCTAATCAAGTTTTTTGGGGTCGAGGTGCCGTAAAGCACTAAATC GGAACCCTAAAGGGAGCCCCCGATTTAGAGCTTGACGGGGAAAGCCGGCGAACGTGG CGAGAAAGGAAGGGAAGAAAGCGAAAGGAGCGGGCGCTAGGGCGCTGGCAAGTGTA GCGGTCACGCTGCGCGTAACCACCACACCCGCCGCGCTTAATGCGCCGCTACAGGGCG CGTCCATTCGCCATTCAGGCTGCGCAACTGTTGGGAAGGGCGATCGGTGCGGGCCTCT TCGCTATTACGCCAGCTGGCGAAAGGGGGATGTGCTGCAAGGCGATTAAGTTGGGTA ACGCCAGGGTTTTCCCAGTCACGACGTTGTAAAACGACGGCCAGTGAATTGTAATAC GACTCACTATAGGGCGAATTGGGCCCTCTAGATGCATGCTCGAGCGGCCGCCAGTGT GATGGATCTGAAGTAAATTATCCATTATCCATTCCCGTTTCTCTAATTATGGGTAAT GGAGTGTGAAGGGGACACAGTAATCCCTGAAATCATGTTTACTTCAGCGGAGCCGCC CCTTGGTGATGGGGGAGGGGAGGCACCTGCCAGCGCCAGCAGCTCTGACACTTTTCCG GGGCTATCTGAAAGCTTTCATGAGCCTACAAGAGGAGTGTGACTCTTTCGTTTATTT GACGTGAGAAATGACATCTTTGTTCACCTTGAGGGAAGACATGAAAAGCGGCCATTG TCGTGTCCTGTCTACTGAAGAGGAACCACAGTCAAGTGTGGTCAGTTGAGCTCATTT CTTTTTAAGACTCTGCTGACCTTCGTTGTTAGACATAAGCTCGCCAGCTTCTTACCTG TCCACACTGGCCCTGATGACCCACGACACACATGCAGCCTCAAGCCCTCCTCCGTAGC TGTGCTTGTGTCCTGCCAGGCTAGTTAGACCTGAACTCCAGGGACCTCCCCCTGAGCA ATGTAGTGATGTGCCTGGCTCAGTAATTAAGCCTAATTATGTTTTATGGCGAGCAGA CATCCCCGCTCTTCATTTCCATTTCTGACAGTTTAGTAATTGATGCCCAAGCTCCTAT CTAGCCCCTGGGCTCAGCCTAAGTGTGTCTGCTTGGCATTTTTAGCTGTGCTGAATGA AAAGGCAAGAGTACATGATCTCGCCTCGCCTCCAGCTGCCAGCCCTGGCTGTTCTGGA ACTCACTCTGTAGACCAGACTGGCCTCAGTCTCCCAAGATCCACTTGCCTCTGCCTAC TGAGCTGGGATTAAAGGTGTGCTGCATGCCGTTTTAAAATATTTGTTCCACTTTTTT AAAAGTTTAGAGTGGCTGGGCAGTGGTGGCACAGGCCTTTTAATCCCAGCACTCGGG AGGCAGAGGCAGGCGGATTTTTGAGTTCGAGGCCAGCCTGGTNTTCCGAGGAGTGAG TTCCAGGGACAGCAATGGATACACAGAGAAACCCTGTCTCAAAAAAAAAAAAAAAAC AAAAGAAAAAAAAAAGGGGGGATGGGGGTTAGGTTTATGGTATTGTTGTGAAATTC AAAGGTAGTTTTTTTAAGTCCTGAGAACTGTTTTTAAAAATGTATGTTTAAAAGTAT ACGTATTGTGTTTGTGTGCCTGCACACTCGCATACAATGTAATGAATGCAATTGCTG TAATAAAAGGCGGATCCCAGAACTTTGGTAAACCCCTAGAATGGTATGTTCTAGATA GTTGAGATACTGTGTTCAATAGTCCCATCTCTCCCTGTCCTTCTTTGGAATGTGGTGA TTCATGACTCAGTCTTTAAAAGTTTAAGGTCCAGCTGATGGCGTATTGCAACACTCT GCTAAATCCTGCCCCTTGCGTCATGCGTGACGGGGACTTCGTCTTGTATTGTTGAATT CTCCCCTTGGTCCCGGCTTCTCTTTTTATGCTCCTTAATGGACACTGCCTTCACTCAC AGTCTGTGTGTAGTTAAGCTTGGCTGGACGTAAACTCCTCTTCAGACCTAATAACTT CGTATAGCATACATTATACGAAGTTATATTAAGGGTTATTGAATATGATCGGAATTA TCGAATTCCCTGCAACAGCTGGCTACAGTCACCAGCAAACTCTCCTGCGTGGTGGGGA GAGCTACCTGTCAGAGCTGGGGCTTAGTGGACAATGAACGAAAGCAGATGGCATTGT GAGCCAGTGGTGTGTGTTCCCTGTTACTGAGTCCTTCTGAAATCCCGGAACTTGTATT TCAGATTGCATCCAAGTATGACCAGCAGGCCGAGGAAGATCTGCGCAACTGGATAGA AGAGGTGACAGGCCTAGGCATTGGCACCAACTTCCAGTTGGGGCTGAAGGACGGCAT CATATTGTGCGAACTCATAAACAAGCTACAGCCAGGCTCTGTGAAGAAAGTCAACGA ATCCTCACTAAATTGGCCCCAGTTGGAGAATATCGGCAACTTCATTAAAGCTATCCA GGCTTACGGTATGAAGCCCCATGATATATTTGAAGCAAACGACCTCTTTGAGAATGG CAACATGACCCAGGTTCAGACGACGCTGGTGGCTCTAGCAGGTCTGGCGAAAACAAA AGGATTCCATACAACCATTGACATTGGCGTTAAGTATGCAGAAAAACAAACAAGACG TTTTGATGAAGGCAAATTAAAGGCTGGCCAGAGTGTAATTGGTTTACAGATGGGTAC 
CAACAAATGTGCCAGCCAGGCGGGCATGACAGCCTATGGGACTCGGAGGCATCTTTA TGATCCCAAGATGCAGACGGACAAACCCTTTGACCAGACCACGATTAGCCTGCAGAT GGGCACCAACAAAGGGGCCAGCCAGGCTGGGATGTTAGCACCGGGCACCAGAAGAGA CATCTATGACCAGAAGCTGACATTACAGCCAGTGGACAACTCGACCATTTCTCTACA GATGGGCACCAACAAAGTTGCTTCCCAGAAAGGAATGAGCGTGTATGGGCTTGGGCG GCAAGTATATGACCCCAAGTACTGTGCCGCACCCACAGAACCTGTCATTCACAACGGA AGCCAGGGCACGGGCACCAATGGGTCGGAAATCAGTGATAGCGATTATCAGGCAGAA TACCCCGATGAATATCATGGCGAGTACCCAGACGACTACCCTCGGGAGTACCAGTAT GGCGACGACCAGGGCATTGATTATTTACCGGTCGCCACCATGGTGAGCAAGGGCGAG GAGCTGTTCACCGGGGTGGTGCCCATCCTGGTCGAGCTGGACGGCGACGTAAACGGCC ACAAGTTCAGCGTGTCCGGCGAGGGCGAGGGCGATGCCACCTACGGCAAGCTGACCCT GAAGTTCATCTGCACCACCGGCAAGCTGCCCGTGCCCTGGCCCACCCTCGTGACCACC CTGACCTACGGCGTGCAGTGCTTCAGCCGCTACCCCGACCACATGAAGCAGCACGACT TCTTCAAGTCCGCCATGCCCGAAGGCTACGTCCAGGAGCGCACCATCTTCTTCAAGGA CGACGGCAACTACAAGACCCGCGCCGAGGTGAAGTTCGAGGGCGACACCCTGGTGAA CCGCATCGAGCTGAAGGGCATCGACTTCAAGGAGGACGGCAACATCCTGGGGCACAA GCTGGAGTACAACTACAACAGCCACAACGTCTATATCATGGCCGACAAGCAGAAGAA CGGCATCAAGGTGAACTTCAAGATCCGCCACAACATCGAGGACGGCAGCGTGCAGCT CGCCGACCACTACCAGCAGAACACCCCCATCGGCGACGGCCCCGTGCTGCTGCCCGAC AACCACTACCTGAGCACCCAGTCCGCCCTGAGCAAAGACCCCAACGAGAAGCGCGATC ACATGGTCCTGCTGGAGTTCGTGACCGCCGCCGGGATCACTCTCGGCATGGACGAGCT GTACAAGTAAGTCGAGCCTCTAGAACTATAGTGAGTCGTATTACGTAGATCCAGACA TGATAAGATACATTGATGAGTTTGGACAAACCACAACTAGAATGCAGTGAAAAAAAT GCTTTATTTGTGAAATTTGTGATGCTATTGCTTTATTTGTAACCATTATAAGCTGCA ATAAACAAGTTAACAACAACAATTGCATTCATTTTATGTTTCAGGTTCAGGGGGAGG TGTGGGAGGTTTTTTAATTCGCGGCCGCCAGTGTGATGGATGACGGTATCGATAAGC TTGATCCTTAATTAAGGAGAGGTTTTCACCGTCATCACCGAAACGCGCGAGGCAGCC TCGAGGGAGCTTCAAAAGCGCTCTGGAAGTTCCTATACTTTCTAGAGAATAGGAACT TCGGAATAGGAACTTCAAGATCCCCCTGGCGAAAGGGGGATGTGCTGCAAGGCGATT AAGTTGGGTAACGCCAGGGTTTTCCCAGTCACGACGTTGTAAAACGACGGCCAGTGA ATTCGAGCTCTCCCATATGGTCGAGCAGTGTGGTTTTGCAAGAGGAAGCAAAAAGCC TCTCCACCCAGGCCTGGAATGTTTCCACCCAATGTCGAGCAGTGTGGTTTTCAAGAGG AAGCAAAAAGCCTCTCCCACCCAGGCCTGGAATGTTTCCACCCAATGTCGAGCAAACC CCGCCCAGCGTCTTGTCATTGGCGAATTCGAACACGCAGATGCAGTCGGGGCGGCGCG GTCCCAGGTCCACTTCGCATATTAAGGTGACGCGTGTGGCCTCGAACACCGAGCGAGC GACCCTGCAGCCAATATGGGATCGGCCATTGAACAAGATGGATTGCACGCAGGTTCT CCGGCCGCTTGGGTGGAGAGGCTATTCGGCTATGACTGGGCACAACAGACAATCGGC TGCTCTGATGCCGCCGTGTTCCGGCTGTCAGCGCAGGGGCGCCCGGTTCTTTTTGTCA AGACCGACCTGTCCGGTGCCCTGAATGAACTGCAGGACGAGGCAGCGCGGCTATCGT GGCTGGCCACGACGGGCGTTCCTTGCGCAGCTGTGCTCGACGTTGTCACTGAAGCGGG AAGGGACTGGCTGCTATTGGGCGAAGTGCCGGGGCAGGATCTCCTGTCATCTCACCT TGCTCCTGCCGAGAAAGTATCCATCATGGCTGATGCAATGCGGCGGCTGCATACGCTT GATCCGGCTACCTGCCCATTCGACCACCAAGCGAAACATCGCATCGAGCGAGCACGTA CTCGGATGGAAGCCGGTCTTGTCGATCAGGATGATCTGGACGAAGAGCATCAGGGGC TCGCGCCAGCCGAACTGTTCGCCAGGCTCAAGGCGCGCATGCCCGACGGCGAGGATCT CGTCGTGACCCATGGCGATGCCTGCTTGCCGAATATCATGGTGGAAAATGGCCGCTTT TCTGGATTCATCGACTGTGGCCGGCTGGGTGTGGCGGACCGCTATCAGGACATAGCG TTGGCTACCCGTGATATTGCTGAAGAGCTTGGCGGCGAATGGGCTGACCGCTTCCTCG TGCTTTACGGTATCGCCGCTCCCGATTCGCAGCGCATCGCCTTCTATCGCCTTCTTGA 
CGAGTTCTTCTGAGGGGATCGGCAATAAAAAGACAGAATAAAACGCACGGGTGTTGG GTCGTTTGTTCGGATCCGTCGAGGGAGCTTCAAAAGCGCTCTGAAGTTCCTATACTT TCTAGAGAATAGGAACTTCGGAATAGGAACTTCAAGATCCCCCTGGCGAAAGGGGGA TGTGCTGCAAGGCGATTAAGTTGGGTAACGCCAGGGTTTTCCCAGTCACGACGTTGT AAAACGACGGCCAGTGAATTCGAGCTCTCCCATATGGTCGAGACGGTATCGATAAGC TTGAGATCCTAAGCTTGGCTGGACGTAAACTCCTCTTCAGACCTAATAACTTCGTAT AGCATACATTATACGAAGTTATATTAAGGGTTATTGAATATGATCGGAATTATCGAA TTCCTGCAGCCCATCTGCAGAATTCGCCCTTCTCGGAGGCATCTTTATGATCCCAAGA TGCAGACGGACAAACCCTTTGACCAGACCACGATTAGCCTGCAGATGGGCACCAACA AAGGGGCCAGCCAGGTAAGCGGGGTCTTTTTAACACTTGAAGCAAAGGGCGCGGAAG GGTATGCTAAGTTAGTGCTGACCCAATAGTGAGTAGCTGGACTCTCAAATGGTATTT CTTCCATGGTATTTTTAGTCTTCAGGAAAGTAGATTTTGCTGAGAGAGCAAAATAAT CAAAGGGCCCTTTCTGCAGCCTAAAAAGTGTAGCGTGACTAATGGTTTGGTTGGCAG TAAGATCAGCTGGCCTTTCTGCCTAACCAGAAGAAAAAAGTGATGTTTATATATGTT CCTGCTCCAAAAGCCAAGGAAAGGATAGTTGGTTCTCCCCTGCCCCATTTTGGTTTTG TTTTTAAAGGGGCCCATTTAGGTGGATATGTAAGATTATTTGGCTGTTTGGTAAGAG CTGTTTAATCAGAATTTTTATGAAACATTACGGTAGCAGAAATGAATTCATATGAAA TGGTAGTTAAATAATTTTTAAAGTAGTGGGGAGGAAAGGGTTTGTTCGGCTTACGTT TCCATGCCATAGGCCAACCGAGGCAGGAACCTGGAGGCAGGAGCTGAAGCAGAGAGG AGTGTCACCTGCTGGCTTCCCTTGCTTGCTCTGCTACCGTTCTTAGACAGCCAGGCCT ACCTGCCTGGGAGCCTCCAGCATCCATTAGCACTTCAGAAAATGCCCCACAGACTCTC GCACAGGCCAATCTGATAGAGCTAATTATTTCATTGAGGTCTACAGTTCCCAGGTGT GTCTACCTGAAAGCCAAAATTAGTCACCACAGGTTTTGAGATAGATGAGGGTGTGGA CACGATGGGGGAGAGAGGGACAGGCCGGGTAAAGGCGGTTATCCGGTGGCTTGAGTT TGATCCCTGGAACCCATGAAGACGGAAGGAGAGGGCGACTCTACAACATTGTTCTTC AGCCTTCACATTCACATAACCCTGGACTCTGGACCTAGGTTTGTCTAGTGCCCTCTCC ACTCCTCCСCACСCССACACACAAATAGTAAAAACAGTGAAATACCAGGAATTGTTT GAAAGCATCAGGATTTTATATCTAAAAATCGTGGGACGGAGTTTTGGTAAGCTTTCC TGTTTTTCCAGTGACATCTGTCGTTCCCCGTGTGGAGCGGAGCGCTCCGGGTCACCTG CAGTTGGTTCTCTTGGCGGTCTCTCGTGTTTGCCTTTTCACTCACTCACCTCTCAAGT GTGTGCTTGACAGCAGTGGGTGCTGAGGTGTGGGTTACCCACTGTGAAAGGGAGAGA GGAGCAGGTCTGAGGTGGGACTGTGGGTGGGGGAGATCTTGTGTGGCAGCCTCTTGA GAAGACTTGGTCCCTGAGATCTGCTCAGCCTGGTCTTGTTGAAGGCATGGATGCTTT TTTACAATTGGAGACCTGGGTGGTCATTAATAGACCATGTTCATATGTAATTCAGCA ATATGTGTAAAAAAATGTAGTTTATCTGATTTTTTTTATGTGTCTAGCTATTTCGCC TGTGTGTATGTATATGAGCCTTGTATATGCCTGCTACCCTTGGAAGTCAGGAATGGA TGGATGCCCTGAAACTGAGTTACAGTTTAGGTGCTGGGAACTGGTCCTGGGTCCTCT GCAAGACAGACAAGTGCTCTTAACCACAGAGCTCTTGTCGCCAGCCCTGGGCTTGGTT TTCCTTTGTCCCTCACTTAGTAGACTGACGTATTGCTTCTGTATTACATATAGGAAG TTAAACTCAAGGTAGGTTAATTTGTCTGACACCATATAGTGATCTTAACAGTGGGCA GAGATTTGAAGGAAGAAGGCCTGGCTCAACTCAGCAGCTCAAGAGCCAACGAATCCC GTCATAAATATGAGTGGTATAAGAATATTTTAGTAAGTTAGGGTCACGTCAGAACCA ATTGAAAGTTTTTATGTTAATGCATTCTGTGTGAATTTAATAATTTAGACAGTGTTA AGAAGTATAGATCTTATCTGTTATCTTACCTTGTGTACCATTATGCTCTAATACCAG GCTGGGATGTTAGCACCGGGCACCAGAAGAGACATCTATGACCAGAAGCTGACATTA CAGCCAGTGGACAACTCGACCATTTCTCTACAGATGGGCACCAACAAAGTTGCTTCCC AGAAAGGAATGAGCGTGTATGGGCTTGGGCGGCAAGTATATGACCCCAAGTACTGTG CCGCACCCACAGAACCTGTCATTCACAACGGAAGCCAGGGCACGGGCACCAATGGGTC GGAAATCAGTGATAGCGATTATCAGGCAGAATACCCCGATGAATATCATGGCGAGTA 
CCCAGACGACTACCCTCGGGAGTACCAGTATGGCGACGACCAGGGCATTGATTATTA GAGTCACACACAGGAGCGCAGTATTTAGTCCATTGTTTTATCCAGTGAGACCCAAGC TAGCCTTGAATAATTCTTCTCTCGTCTTCCTGAAACACTATTATGCTTGTTGTACCTT TAAAGTATGCCTTATGTACATTCCTTTCTCCTTTTCCTGCCTCCTCCCTAAATAGCTG CCTTCTAGTGCTGTAGCAAGGGAGCCCTACTGCATAGCCAGTAACTCGCGTCTGTACC ATGGAAAGGGCGGAACGATTCTCCAGGACAGCCAGCTCTTTCGTTGAAGATCTATCT ATGCATTTTTTTACACTTACACATAAACTGGTATTTTCGAACAATAGGAAACTATTT TTTCTCCTTTTTTTACAGTTTTAGTACGTATCTGGCTTGTATGTGGAAGACTAAAAA GTTGATTTGCTAAATGTGGTCTTTGCCAACTAAAATCTGAGATGCAGCTTTAGACCC TGACACGTGGATGTTCTTCTGCAGTCTTGTCTGCTAAGTTTTAAATAAAGTCATGAT CAGTGTGCATTTGTGATTACATGTGTACTCATTCTTTTCCCAAGCTGACGAGGTCTCT CCCGAGTGGCGCTTCGAAAGGCGTGCATGCAGAAATGGCCGAGGACATGCAGGTTTG GGTGGTGTGCCTGCAGACTTCATTTGTGCCAATGTATTACTGTAGAGTCGCTCTGTT TCCTTCAACTGTATTTATTGCTGCGTTTCTCAAAGGGCGAATTCCAGCACACTGGCGG CCGTTACTAGTGGATCCGAGCTCGGTACCAAGCTTGGCGTAATCATGGTCATAGCTG TTTCCTGTGTGAAATTGTTATCCGCTCACAATTCCACACAACATACGAGCCGGAAGC ATAAAGTGTAAAGCCTGGGGTGCCTAATGAGTGAGCTAACTCACATTAATTGCGTTG CGCTCACTGCCCGCTTTCCAGTCGGGAAACCTGTCGTGCCAGCTGCATTAATGAATCG GCCAACGCGCGGGGAGAGGCGGTTTGCGTATTGGGCGCTCTTCCGCTTCCTCGCTCAC TGACTCGCTGCGCTCGGTCGTTCGGCTGCGGCGAGCGGTATCAGCTCACTCAAAGGCG GTAATACGGTTATCCACAGAATCAGGGGATAACGCAGGAAAGAACATGTGAGCAAAA GGCCAGCAAAAGGCCAGGAACCGTAAAAAGGCCGCGTTGCTGGCGTTTTTCCATAGG CTCCGCCCCCCTGACGAGCATCACAAAAATCGACGCTCAAGTCAGAGGTGGCGAAACC CGACAGGACTATAAAGATACCAGGCGTTTCCCCCTGGAAGCTCCCTCGTGCGCTCTCC TGTTCCGACCCTGCCGCTTACCGGATACCTGTCCGCCTTTCTCCCTTCGGGAAGCGTG GCGCTTTCTCATAGCTCACGCTGTAGGTATCTCAGTTCGGTGTAGGTCGTTCGCTCCA AGCTGGGCTGTGTGCACGAACCCCCCGTTCAGCCCGACCGCTGCGCCTTATCCGGTAA CTATCGTCTTGAGTCCAACCCGGTAAGACACGACTTATCGCCACTGGCAGCAGCCACT GGTAACAGGATTAGCAGAGCGAGGTATGTAGGCGGTGCTACAGAGTTCTTGAAGTGG TGGCCTAACTACGGCTACACTAGAAGAACAGTATTTGGTATCTGCGCTCTGCTGAAG CCAGTTACCTTCGGAAAAAGAGTTGGTAGCTCTTGATCCGGCAAACAAACCACCGCT GGTAGCGGTGGTTTTTTTGTTTGCAAGCAGCAGATTACGCGCAGAAAAAAAGGATCT CAAGAAGATCCTTTGATCTTTTCTACGGGGTCTGACGCTCAGTGGAACGAAAACTCA CGTTAAGGGATTTTGGTCATGAGATTATCAAAAAGGATCTTCACCTAGATCCTTTTA AATTAAAAATGAAGTTTTAAATCAATCTAAAGTATATATGAGTAAACTTGGTCTGAC AGTTACCAATGCTTAATCAGTGAGGCACCTATCTCAGCGATCTGTCTATTTCGTTCAT CCATAGTTGCCTGACTCCCCGTCGTGTAGATAACTACGATACGGGAGGGCTTACCATC TGGCCCCAGTGCTGCAATGATACCGCGAGACCCACGCTCACCGGCTCCAGATTTATCA GCAATAAACCAGCCAGCCGGAAGGGCCGAGCGCAGAAGTGGTCCTGCAACTTTATCC GCCTCCATCCAGTCTATTAATTGTTGCCGGGAAGCTAGAGTAAGTAGTTCGCCAGTT AATAGTTTGCGCAACGTTGTTGCCATTGCTACAGGCATCGTGGTGTCACGCTCGTCGT TTGGTATGGCTTCATTCAGCTCCGGTTCCCAACGATCAAGGCGAGTTACATGATCCCC CATGTTGTGCAAAAAAGCGGTTAGCTCCTTCGGTCCTCCGATCGTTGTCAGAAGTAA GTTGGCCGCAGTGTTATCACTCATGGTTATGGCAGCACTGCATAATTCTCTTACTGTC ATGCCATCCGTAAGATGCTTTTCTGTGACTGGTGAGTACTCAACCAAGTCATTCTGA GAATAGTGTATGCGGCGACCGAGTTGCTCTTGCCCGGCGTCAATACGGGATAATACC GCGCCACATAGCAGAACTTTAAAAGTGCTCATCATTGGAAAACGTTCTTCGGGGCGA AAACTCTCAAGGATCTTACCGCTGTTGAGATCCAGTTCGATGTAACCCACTCGTGCAC CCAACTGATCTTCAGCATCTTTTACTTTCACCAGCGTTTCTGGGTGAGCAAAAACAG 
GAAGGCAAAATGCCGCAAAAAAGGGAATAAGGGCGACACGGAAATGTTGAATACTCA TACTCTTCCTTTTTCAATTCAGAAGAACTCGTCAAGAAGGCGATAGAAGGCGATGCG CTGCGAATCGGGAGCGGCGATACCGTAAAGCACGAGGAAGCGGTCAGCCCATTCGCC GCCAAGCTCTTCAGCAATATCACGGGTAGCCAACGCTATGTCCTGATAGCGGTCCGCC ACACCCAGCCGGCCACAGTCGATGAATCCAGAAAAGCGGCCATTTTCCACCATGATAT TCGGCAAGCAGGCATCGCCATGGGTCACGACGAGATCCTCGCCGTCGGGCATGCGCGC CTTGAGCCTGGCGAACAGTTCGGCTGGCGCGAGCCCCTGATGCTCTTCGTCCAGATCA TCCTGATCGACAAGACCGGCTTCCATCCGAGTACGTGCTCGCTCGATGCGATGTTTCG CTTGGTGGTCGAATGGGCAGGTAGCCGGATCAAGCGTATGCAGCCGCCGCATTGCAT CAGCCATGATGGATACTTTCTCGGCAGGAGCAAGGTGGGATGACAGGAGATCCTGCC CCGGCACTTCGCCCAATAGCAGCCAGTCCCTTCCCGCTTCAGTGACAACGTCGAGCAC AGCTGCGCAAGGAACGCCCGTCGTGGCCAGCCACGATAGCCGCGCTGCCTCGTCCTGC AGTTCATTCAGGGCACCGGACAGGTCGGTCTTGACAAAAAGAACCGGGCGCCCCTGC GCTGACAGCCGGAACACGGCGGCATCAGAGCAGCCGATTGTCTGTTGTGCCCAGTCAT AGCCGAATAGCCTCTCCACCCAAGCGGCCGGAGAACCTGCGTGCAATCCATCTTGTTC AATCATGCGAAACGATCCTCATCCTGTCTCTTGATCAGATCTTGATCCCCTGCGCCAT CAGATCCTTGGCGGCAAGAAAGCCATCCAGTTTACTTTGCAGGGCTTCCCAACCTTAC CAGAGGGCGCCCCAGCTGGCAATTCCGGTTCGCTTGCTGTCCATAAAACCGCCCAGTC TAGCTATCGCCATGTAAGCCCACTGCAAGCTACCTGCTTTCTCTTTGCGCTTGCGTTT TCCCTTGTCCAGATAGCCCAGTAGCTGACATTCATCCGGGGTCAGCACCGTTTCTGCG GACTGGCTTTCTACGTGTTCCGCTTCCTTTAGCAGCCCTTGCGCCCTGAATTTTGTTA AAATTCGCGTTAAATTTTTGTTAAATCAGCTCATTTTTTAACCAATAGGCCGAAATC GGCAAAATCCCTTATAAATCAAAAGAATAGACCGAGATAGGGTTGAGTGTTGTTCCA GTTTGGAACAAGAGTCCACTATTAAAGAACGTGGACTCCAACGTCAAAGGGCGAAAA ACCGTCTATCAGGGCGATGGCCCACTAC

short homology arm: 465-1965

loxP: 2000-2033

Cnn3 (ex2-7)-GFP: 2241-3908

SV40 poly(A): 3946-4080

FRT: 4266-4313

neoR: 4716-5519

HSV TK poly(A): 5526-5574

FRT: 5606-5653

loxP: 5837-5870

long homology arm: 5937-9086 


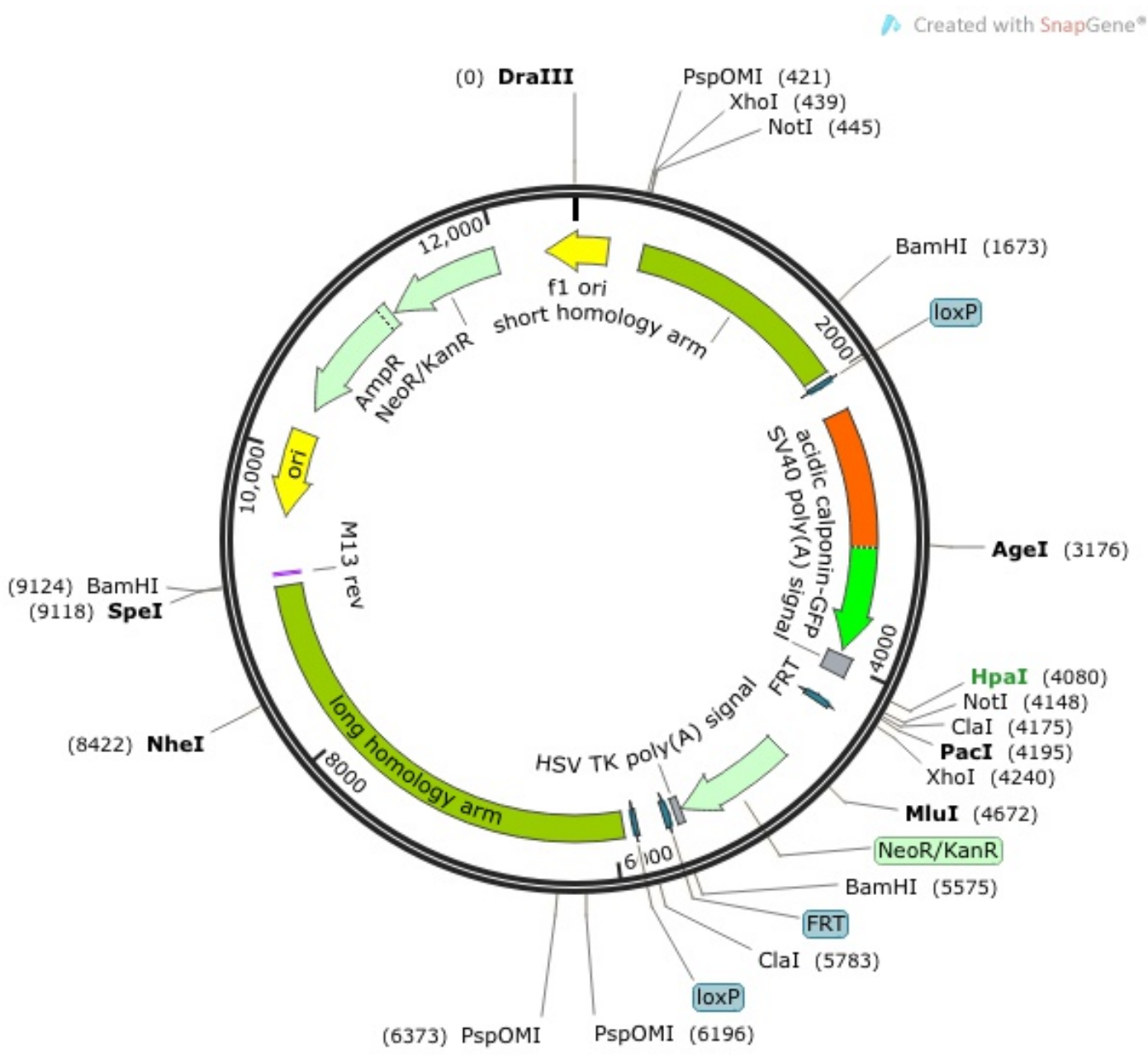

pCR-TOPO calponin 3-GFP_targeting vector

\section{sequence $5^{\prime}$ internal probe}

GGGATGGGGGTTAGGTTTATGGTATTGTTGTGAAATTCAAAGGTAGTTTTTTTAAGT CCTGAGAACTGTTTTTAAAAATGTATGTTTAAAAGTATACGTATTGTGTTTGTGTGC CTGCACACTCGCATACAATGTAATGAATGCAATTGCTGTAATAAAAGGCGGATCCCA GAACTTTGGTAAACCCCTAGAATGGTATGTTCTAGATAGTTGAGATACTGTGTTCAA TAGTCCCATCTCTCCCTGTCCTTCTTTGGAATGTGGTGATTCATGACTCAGTCTTTAA AAGTTTAAGGTCCAGCTGATGGCGTATTGCAACACTCTGCTAAATCCTGCCCCTTGCG TCATGCGTGACGGGGACTTCGTCTTGTATTGTTGAATTCTCCCCTTGGTCCCGGCTTC TCTTTTTATGCTCCTTAATGGACACTGCCTTCACTCACAGTCTGTGTGTAGTTAAGCT TGGCTGGACGTAAACTCCTCTTCAGACCTAATAACTTCGTATAGCATACATTATACG AAGTTATATTAAGGGTTATTGAATATGATCGGA

sequence 3' external probe

ACCAAGATTTACCTGGTCCTTGTTCTACTACAACTTGGCTGCCCTGGGTTTATCTGAA ATTCGATTTGGAATTCTCTGAAACGCAAGGGGGAGTTATTACATGTGTCATGTAAAT 
GATCCTAAAGCCAGGCATGAGGTGTGCTGTTTGATTAGAGCAGGCTAACCCACAGCT AAGTGTGGGAAAGGGCAGTGCTGTCACGGGGGAAGCTGCTCGGTCACACCCTCTGAC CTTGTCTCAGGTCAAGGCTGACTGATGGCCTCGGGTGGTTTTGTAAAGACAGATAAG AGATGTTGAACCCACAGAAAGGATGTATGCTTGTTCCTCCTGTGGTGGCTTGCTTAG ATGCTTCTGGGAGTGCCCTGTAAATCACTGTAGGAGACTGGGAGCTAAATGAATTCT GTGGTACTATTAAAGAAAAAATATCTAAAGTGGTCCTGAGCTGTCAGGCTGTTTCTT G

\section{genotyping protocol by PCR}

primer 1: GGACACTGCCTTCACTCACA

primer 2: $\quad$ CCGTCCTTCAGCCCCAA

primer 3: TTGTTGGTGCCCATCTGCA

Primer 1 and 2 discriminate between the floxed (PCR product of $417 \mathrm{bp}$ ) and the wildtype allele ( $310 \mathrm{bp}$ ). Primers 1 and 3 discriminate between the floxed (888 $\mathrm{kb})$ and the deleted allele (254 bp). 